\title{
TURISMO DE CRUCEROS EN BARCELONA. DE LA MARGINALIDAD AL LIDERAZGO INTERNACIONAL
}

\author{
Luis Alfonso Garay Tamajón \\ Estudios de Economía y Empresa. Universitat Oberta de Catalunya \\ Igaray@uoc.edu \\ Gemma Cànoves Valiente \\ Departamento de Geografía. Universitat Autònoma de Barcelona \\ gemma.canoves@uab.cat
}

\section{RESUMEN}

Este artículo presenta el éxito del turismo de cruceros en la ciudad de Barcelona. Tras ocupar una posición de marginalidad durante décadas, esta tipología ha pasado a ser uno de los símbolos del desarrollo turístico de la ciudad, especialmente a partir de la celebración de los Juegos Olímpicos de Barcelona. Demostraremos como la conjunción de una correcta promoción por parte del consorcio turístico Turisme de Barcelona y las continuas inversiones en las infraestructuras portuarias de la ciudad en relación al tráfico crucerista se encuentran tras esta experiencia exitosa.

Palabras clave: Turismo de Cruceros, Barcelona, Infraestructuras, Marca de Destino.

\section{ABSTRACT}

This paper presents the case study of cruise tourism in the city of Barcelona. From a position of marginalization for decades, this type has become a symbol of tourism development in the city, especially after the conclusion of the Olympic Games in Barcelona. We demonstrate how the combination of proper promotion by the Turisme de Barcelona

Fecha de recepción: febrero 2011.

Fecha de aceptación: julio 2012. 
Consortium and the continuous investments on cruiser traffic in the port city are behind this success story.

Key words: Cruise Tourism, Barcelona, Infrastructures, Destination Branding.

\section{INTRODUCCIÓN}

En la actualidad Barcelona es el principal puerto europeo de cruceros y se sitúa a nivel mundial entre el cuarto y el quinto destino en esta tipología. Con cerca de dos millones y medio de pasajeros llegados en el año 2010 y con un incremento de este flujo de cerca de un $9 \%$ interanual en plena etapa de crisis económica, las perspectivas son que éste producto sostenga o incluso aumente su crecimiento a lo largo del año 2011. Sin embargo, este considerable desarrollo es relativamente reciente, aunque la actividad crucerista haya existido en la ciudad desde hace tiempo.

Este artículo analiza el éxito del turismo de cruceros en la ciudad de Barcelona en las últimas tres décadas. El segmento crucerista ha pasado de una situación en la que su existencia era prácticamente marginal a otra en la que Barcelona se ha situado como el principal destino de turismo de cruceros en el Mediterráneo y uno de los principales a nivel mundial. Para comprender esta considerable progresión analizaremos las diferentes dinámicas que la han propiciado. Entre éstas, el despegue de Barcelona como uno de los destinos turísticos urbanos más importantes a nivel internacional tras las Olimpiadas de 1992, el auge del turismo de cruceros y sus mercados a nivel internacional en los últimos años y muy especialmente la intervención conjunta (y acertada) del sector público y privado de la ciudad para potenciar este sector en Barcelona.

\section{REVISIÓN DE LA LITERATURA}

La literatura académica respecto al turismo de cruceros es todavía muy escasa y más lo es en el caso de Barcelona, a pesar de tratar con uno de los principales fenómenos turísticos de los últimos años en este destino. De hecho, entre los primeros estudios a nivel internacional cabe destacar algunos a finales de la década de los ochenta (Lawton y Butler, 1987) y especialmente ya en la década de los noventa (Dwyer and Forsyth, 1996, 1998; Hall and Braithwaite, 1990), principalmente centrados en el mercado norteamericano y el destino caribeño, que hasta entonces habían sido absolutamente predominantes. También en la misma época comenzaban a escribir en España autores como Esteve (1998). En los siguientes años, diferentes referencias aportarían nuevos puntos de vista al tema (Butler, 1999; Capacci, 2000; Pérez, 1999; Wood, 2000; Yepes et al., 2000), con lo que la literatura al respecto se ampliaría gradualmente. Volviendo al caso español, quizás una de las referencias más interesantes ha sido la tesis doctoral de Murias (2002), que aunque centrada en aspectos de infraestructura y tecnología portuaria, ofrecía también una interesante visión de la evolución de la oferta y la demanda del sector. En los años siguientes, nuevas referencias han ampliado la literatura del crucerismo, ya sea en forma de artículos en revistas científicas (Andriotis and Agiomirgianakis, 2010; Brida and Zapata-Aguirre, 2008; Brida and Zapata, 
2010; García, 2008; Johnson, 2002; Kester, 2003; Marusic et al., 2008; Mazorra, 2005; Weaver, 2005), como también por lo que respecta a los estudios realizados por Manuel Butler desde el mundo académico (Butler, 2001) y para la Organización Mundial del Turismo en la última década (OMT, 2003; OMT, 2008). Para el caso de España es muy valiosa una referencia contemporánea a la presente, la de Brida y otros (2012), que estudia las percepciones de los residentes sobre este turismo en Cartagena.

En dicha literatura se observa la predominancia, prácticamente total, de las referencias relacionadas con el caso del Caribe, destino tradicional del turismo de cruceros y del mercado norteamericano, que hasta la primera década del presente siglo ocupaba y movilizaba a un $90 \%$ de la demanda. No en vano, las principales compañías del mismo siguen siendo norteamericanas. En este sentido, los temas estudiados han sido variados, especialmente centrados en la oferta (Lawton y Butler, 1987), observando los procesos de concentración e internacionalización (Hall y Braithwaite, 1990; Wood, 2000), el impacto económico y social en los destinos de este negocio (Braun, 2002; Brida and Zapata-Aguirre, 2008; Brida and Zapata, 2010; Dwyer and Forsyth, 1996, 1998), las implicaciones medioambientales de su desarrollo (Johnson, 2002) y la percepción cambiante de la demanda (Andriotis y Agiomirgianakis, 2010). Sin embargo, es importante notar la escasez de estudios para el caso del Mediterráneo, lo que en parte tiene su explicación debido a que su despegue ha sido reciente y su peso en relación a otros destinos resulta incipiente (Drisdale, 1997). En esta dinámica, Barcelona, como el principal puerto base de la región, es el claro ejemplo de la explosión del turismo de cruceros en las dos últimas décadas.

\section{METODOLOGÍA}

Al respecto de la metodología utilizada hemos tenido en cuenta la literatura anteriormente presentada, especialmente por lo que respecta a las referencias de la OMT en la última década. En todo caso, la escasez o ausencia de trabajos al respecto del crucerismo en Barcelona nos ha obligado a recurrir a otras referencias. Algunas de ellas se relacionan directamente con la evolución de los aspectos clave que ha considerado el análisis, como el impulso de la promoción turística en la ciudad (Duran, 2002), la evolución de las infraestructuras portuarias (Alemany, 2002) y el desarrollo de la demanda crucerista (Paniagua, 2005). Para éste último aspecto, a parte de los comentarios y análisis presentados en informes como el de la OMT o el European Cruise Council, se han tenido en cuenta las estadísticas relativas a esta tipología que ofrece la Autoritat Portuaria de Barcelona (APB, 2006-2009) desde hace unos años. En todo caso, conviene siempre ser cautos con la información facilitada al respecto del número de personas que visitan los destinos gracias al turismo de cruceros, ya que suelen existir confusiones al distinguir entre pasajeros y cruceristas. En este sentido y como ejemplo, mientras los pasajeros embarcan en un puerto con el fin de trasladarse (por el motivo que fuere) a un puerto de destino, los cruceristas embarcan con el objetivo de realizar un itinerario y por motivos vacacionales. Este aspecto se ha tenido en cuenta en el momento de presentar los datos referentes a la demanda.

La estructura de este trabajo es la siguiente: se inicia con una presentación de la evolución del turismo de cruceros a nivel internacional, que sirve para situar al lector en contexto y a continuación se realiza un breve recorrido por los antecedentes del mismo en Barcelona. 
El siguiente apartado, central en nuestro análisis, muestra el impacto de los Juegos Olímpicos de 1992 en esta tipología, ya que sin dicho evento sería imposible entender el desarrollo del turismo en la ciudad de forma general y del crucerismo en particular. Posteriormente, los últimos apartados muestran la explosión del fenómeno crucerista, ya sea en la última década como en la actualidad. El trabajo finaliza con las principales conclusiones y líneas de trabajo propuestas de cara al futuro.

\section{UNA SÍNTESIS DE LA EVOLUCIÓN HISTÓRICA DEL TURISMO DE CRUCEROS}

Debido a su despegue reciente, y de la misma forma que sucede con otras tipologías turísticas, el turismo de cruceros suele considerarse como un producto turístico novedoso, lo que es en parte equivocado. De hecho, el turismo de cruceros, al igual que otros productos originados antes del turismo de masas, es una de esas tipologías que representa bien la transformación de la actividad turística a partir de la recuperación de fenómenos que renacen tras una crisis que se prolonga décadas y que sucede a una primera etapa de esplendor, justo en la época en la que el turismo nacía como actividad de ocio moderna. De hecho, Murias (2002) sitúa su origen en la década de los treinta y cuarenta del siglo XIX, cuando por ejemplo aparecen en los diarios de la ciudad alemana de Hamburgo las primeras ofertas de viaje relacionadas con este tipo de turismo. Obviamente se trataba de una actividad de ocio elitista, reservada a grandes fortunas y de hecho, sería este tipo de demanda la que enriqueció a emprendedores como Thomas Cook y facilitó la consolidación de su compañía en una de las primeras agencias de viajes (Towner, 1985). Poco después de que Cook iniciara sus famosos cruceros a Egipto, entraba en liza otra de las grandes compañías navieras a nivel internacional, la compañía británica Peninsular and Oriental Steam Ship Navigation Company, más conocida por $\mathrm{P} \& \mathrm{O}$, cuyos primeros cruceros se iniciarían hacia 1858.

De hecho, (Fernández Fúster, 1991) en su tratado clásico sobre la historia del turismo consideraba que el período inaugural del turismo de cruceros transcurre entre mediados del siglo XIX y el estallido de la Primera Guerra Mundial, cuando las compañías navieras comienzan a interesarse por este nuevo negocio, como en el caso de la misma $P \& O, C$ unard o White Line (la naviera del famoso Titanic). Su éxito se basaba en la reproducción de un fenómeno recurrente en el turismo (Buzard, 1993): el mimetismo en las actividades vinculadas al ocio y el turismo, ya que una gran parte de estos primeros viajes en crucero reproducían el itinerario que habían seguido décadas antes las corrientes de viajeros aristócratas del Grand Tour, con destinos como Italia, Grecia o Egipto, ahora accesibles a una nueva clase, la burguesa. El crucero era el medio perfecto para compatibilizar las necesidades de estos clientes con el desplazamiento a diferentes destinos exóticos.

Tras esta primera etapa inaugural, una nueva era se iniciaría tras el fin de la Primera Guerra Mundial, cuando la crisis de la navegación transatlántica derivada de la caída del transporte de inmigrantes, obligaría a muchas compañías a transformar sus buques de línea en cruceros (Murias, 2002). En gran medida el anterior y el presente período se pueden considerar como una primera etapa dorada del turismo de cruceros, aunque seguiría estando reservado durante el periodo de entreguerras a una demanda muy restringida, de alto nivel adquisitivo. Finalmente, el estallido de la Segunda Guerra Mundial interrumpiría y transformaría su desarrollo, ya que la mayor parte de los buques serían destinados al transporte de 
tropas o servicios logísticos y la mayoría serían hundidos o fuertemente dañados durante el conflicto. La inmediata posguerra no ofrecía tampoco un contexto favorecedor, con una aviación todavía incipiente y escasos medios para impulsar una actividad turística tanto desde la perspectiva de la oferta como por el impulso de la demanda.

La situación cambiará notablemente a partir de los años sesenta, especialmente a causa del desarrollo de la aviación comercial y de la irrupción en el tráfico aéreo transatlántico del Boeing 747 de la compañía Pan Am, más conocido como Jumbo, que con sus más de 400 pasajeros transformaría totalmente el mundo del transporte a larga distancia y condenaría definitivamente a las compañías navieras transatlánticas, ya afectadas por las restricciones en la entrada de inmigrantes en los Estados Unidos y otros países. Es a partir de este momento, cuando las compañías se vieron forzadas a buscar nuevos mercados para sobrevivir. Si la dificultad estribaba en la adaptación de los buques de línea en centros de ocio con todo tipo de servicios para el turista, la respuesta (especialmente en el mercado norteamericano) fue hábil y se centró en ofrecer precios mucho más reducidos facilitando el acceso de un amplio segmento de la población. Este fue el inicio de la popularización definitiva de dicha tipología y dio paso a las principales compañías modernas como Carnival, Royal Caribbean Cruise Line o Princess.

En este sentido, uno de los principales avances responde a la estrategia de estas compañías para vender el buque como el principal destino, o conseguir que el buque sea el «resort», junto con el atractivo de los lugares que se visitan durante el viaje. Además, las compañías navieras se fueron adaptando al crecimiento de la demanda con nuevos y mayores naves que ofrecían un mayor número de servicios al turista. Con el crecimiento de los buques se facilita la transformación del producto y los cruceros pasan de ofrecer la tradicional fórmula del turismo de masas, el «all inclusive», es decir ofrecer prácticamente todos los servicios que el cliente requiere en el mismo barco y que se pagan de forma conjunta, a añadir un conjunto de servicios extra que se pagan por separado. Además, las compañías deben estar más preparadas en otros aspectos, como la seguridad de los pasajeros y la tripulación.

En todo caso, el incremento de la capacidad permite captar el crecimiento de la demanda y generar mayores rentabilidades e ingresos a bordo derivados del efecto escala, pero también hay que tener en cuenta que el tamaño de las naves puede venir limitado por factores comerciales y económicos: el riesgo de asumir altas inversiones en un solo buque es muy elevado y al alcance de pocas compañías. Así, la concentración está en consonancia con la capacidad para disponer de buques con una oferta flexible, que permita a las compañías operar en diferentes contextos o concentrarse en destinos muy competitivos. De hecho, el gigantismo en el sector del crucero está realmente al alcance de muy pocas empresas, especialmente los tres grandes grupos que dominan el sector como Carnival Corporation, Royal Caribbean y Star Cruises. Finalmente, también es conveniente reconvertir la imagen de los cruceros y el efecto disuasorio de ofrecer un producto cada vez más masificado. La flexibilidad debe también venir por la capacidad para poder segmentar a los clientes en diferentes grupos, a los que se les pueden ofrecer diferentes servicios.

Por otro lado, el papel de las compañías también fue decisivo para impulsar la aparición de las primeras asociaciones regionales, como la Cruise Lines International Association en América o la Cruise Europe en Europa, cuyo papel será clave para canalizar la relación entre compañías y puertos y la cooperación entre estos últimos (Murias, 2002). En este mismo 
sentido, la adaptación de las infraestructuras portuarias fue decisiva para el desarrollo de este tipo de tráfico. Finalmente, la globalización ha tenido también un impacto decisivo en la oferta crucerista, ya sea a través del proceso de internacionalización como especialmente en la creciente concentración, lo que ha reducido el número de operadores y ha incrementado su poder de negociación.

En relación a la concentración de la oferta cabe decir que en la actualidad tres grandes grupos (Carnival Corporation, Royal Caribbean y Star Cruises) controlan el $88 \%$ de la oferta mundial y se presentan como grandes compañías multimarca (OMT, 2008). La mayor parte de las compañías se inclina en los últimos años por ampliar la oferta en los segmentos de renta media, que son los que más están creciendo, y además se inicia un importante proceso de especialización en los itinerarios y los tipos de buque, y en cuanto a los mercados crece el interés por lo temático y especialmente por el mercado familiar. El crecimiento de la oferta se refleja también en el hecho de que las principales navieras se sitúan entre las primeras empresas turísticas respecto a la capitalización bursátil y la tasa de rentabilidad. De hecho, la oferta de turismo de cruceros alcanzaba en 2006 las 300.000 camas (OMT, 2008), el equivalente a la mitad de la capacidad hotelera de una región turística tan importante como el Sudeste Asiático, lo que nos muestra la relevancia del volumen alcanzado.

Tabla 1

MATRIZ DE ORIGEN (MERCADOS) Y DESTINO (REGIONES) DEL TURISMO DE CRUCEROS A NIVEL INTERNACIONAL. 2005 Número de pasajeros en cruceros por origen (mercados) y regiones (destinos)

\begin{tabular}{|l|c|c|c|c|c|c|c|}
\hline \multicolumn{1}{|c|}{ Origen/ } & Caribe & $\begin{array}{c}\text { Medite- } \\
\text { rráneo }\end{array}$ & $\begin{array}{c}\text { Norte de } \\
\text { Europa }\end{array}$ & Alaska & $\begin{array}{c}\text { Sudamé- } \\
\text { rica }\end{array}$ & $\begin{array}{c}\text { Asia- } \\
\text { Pacífico }\end{array}$ & Total \\
\hline \multicolumn{1}{|c}{ Destino } & & & & & & & \\
\hline Reino Unido & 300 & 467 & 190 & 22 & 12 & 7 & 998 \\
\hline Alemania & 125 & 314 & 192 & - & 20 & - & 651 \\
\hline Italia & 48 & 423 & 42 & - & - & - & 513 \\
\hline España & 51 & 311 & 17 & - & - & - & 379 \\
\hline Francia & 55 & 152 & 26 & - & - & - & 233 \\
\hline Resto de Europa & 78 & 268 & 34 & - & - & - & 380 \\
\hline EEUU y Canadá & 6.713 & 1.822 & 1.033 & 858 & 294 & 132 & 10.852 \\
\hline Japón & 18 & 10 & - & 20 & - & 75 & 123 \\
\hline TOTAL & 7.388 & 3.767 & 1.534 & 900 & 326 & 214 & $\mathbf{1 4 . 1 2 9}$ \\
\hline
\end{tabular}

Fuente: OMT (2008)

Por otro lado, aunque el turismo de cruceros puede entenderse como una tipología de resort en constante movimiento, está demostrado su destacado impacto en los principales destinos en los que realiza sus escalas. La Tabla 1 muestra que el principal destino en la actualidad sigue siendo el Caribe especialmente durante la época invernal, donde recibe gran parte de la demanda norteamericana, que sigue siendo con diferencia la más importante en todo el mundo, aunque debido a su situación de consolidación su crecimiento es bas- 
tante lento (hacia un 3\% interanual a mediados de la los noventa). Como se puede apreciar, el Mediterráneo es el destino que más ha crecido en los últimos años (entre mediados de los noventa y mediados del 2000 prácticamente dobló la demanda captada), y se sitúa en segunda posición pero todavía a distancia del Caribe, con una demanda que se reparte entre los mercados europeo y norteamericano.

A continuación les siguen, también a distancia, la Europa Atlántica, Alaska, Sudamérica y Asia-Pacífico. En general, el turismo de cruceros sigue unas pautas muy similares a las de los destinos terrestres en relación a los principales mercados captados, que siguen siendo regionales. Ésta es una de las razones por las cuales destinos como Asia-Pacífico tienen un menor crecimiento que otros, ya que la demanda regional sigue siendo relativamente baja en comparación a otros mercados, como el estadounidense. Finalmente es interesante observar que la mayor parte de macro-destinos del turismo de cruceros tienen un comportamiento estacional que permite a las navieras diversificar sus operaciones y mejorar la rentabilidad de sus activos, utilizando la estrategia de operar en verano en el Mediterráneo y en el Caribe en Invierno (Castejón, 1998).

Desde la perspectiva de la demanda, con la definitiva popularización de este tipo de turismo e incluso una masificación en según qué destinos, el crecimiento medio en las últimas décadas ha sido espectacular, cercano al 8\%, con cerca de 15 millones de turistas en 2005 (ver Tabla 1). Es sin duda alguna una de las tipologías protagonistas de la transformación del turismo contemporáneo. El principal mercado que ha conformado esta demanda ha sido el norteamericano, con Miami como puerto base emblemático y con el Caribe como principal destino de las escalas de los cruceros. Europa es el siguiente mercado en importancia, aunque a distancia del estadounidense. En todo caso, se aprecian en esta tipología distribuciones muy similares a las que se producen en el turismo en general, con diferentes macro-regiones a nivel internacional en la que se concentran mercados y destinos. Aun así, el crecimiento del mercado norteamericano en Europa y del número de europeos que realizan cruceros en el Caribe también ha sido creciente en los últimos años. En todo caso, el turismo de cruceros sigue experimentando una demanda creciente y aún se considera que es una tipología con un enorme potencial, ya que existen mercados que todavía tienen mucho recorrido (especialmente Asia). En este sentido, la Cruise Line International Association (CLIA, 2005) estima un mercado potencial de cerca de 30 millones de turistas en los siguientes años.

Por otro lado, al respecto del perfil de esta demanda la OMT (2008) señala un alto número de pasajeros primerizos, que realizan un viaje en crucero por primera vez. Además, y a pesar de la idea generalizada, no se trata de un viajero mayoritariamente de la tercera edad, su edad media se sitúa alrededor de los 49 años (y tiende a bajar), de ingresos altos, generalmente en pareja (más de un 80\%), con educación universitaria (cerca de un 60\%) y empleados a tiempo completo (también cerca de un 60\%). El turismo de cruceros puede además vincularse fácilmente con las nuevas tendencias de la demanda, pues puede ofrecer en poco tiempo una experiencia de viaje interesante y variada. Es en este sentido, que uno de los principales intereses de la oferta se centra recientemente en poder ofrecer trayectos cortos (de 3 a 4 días), más especializados y adaptados a las disponibilidades de tiempo libre de la sociedad actual, abaratando además el paquete contratado y pudiendo acceder así a un mayor segmento de demanda. 
En definitiva, todos los indicadores sitúan al turismo de cruceros como uno de los negocios con mejor presente y mayores perspectivas de futuro en los próximos años. Muchos son los destinos que han realizado una importante apuesta por él en los últimos años. Y entre ellos se encuentra Barcelona, que es a su vez una de las principales plazas de turismo urbano en Europa. El desarrollo de la ciudad como destino de cruceros es dilatado en el tiempo pero su posición entre los principales destinos es relativamente reciente. En los siguientes apartados vamos a analizar cómo se ha producido esta evolución.

\section{EL TURISMO DE CRUCEROS EN LA BARCELONA PRE-OLÍMPICA}

El espectacular despegue de Barcelona como destino del turismo de cruceros es relativamente reciente, pero su historia es dilatada. Se tiene noticia de la creación de las primeras navieras que operaban en el puerto de Barcelona ya a inicios del siglo XIX (Ybarra, creada en 1823), aunque durante todo este siglo predominaran los desplazamientos de viajeros por motivos lejanos al ocio y prácticamente siempre en relación al desplazamiento de emigrantes desde la Península y especialmente en dirección a las colonias y países independientes del ámbito latinoamericano (un aspecto en el que no difiere de otros puertos europeos en esta época). Hacia finales del mismo siglo, la labor de la Junta de Obras del Puerto de la ciudad daría lugar al primer muelle trasversal y por los mismos años empezaría a funcionar la compañía Transatlántica (1881) y ya a principios del siglo XX la Transmediterránea (1917), que absorbía a anteriores compañías como la Marítima de Barcelona (Murias, 2002). Estas navieras generalmente cubrían líneas regulares y entre sus viajeros se encontraban básicamente emigrantes aunque también los primeros viajeros que se desplazaban por mar en primera clase y por motivos de ocio. La diferencia entre la primera clase y las demás (segunda y tercera) era en esta época muy grande y los servicios de primera eran realmente lujosos aunque el número de pasajeros, en contraste con las otras dos, era muy escaso. A diferencia de otros países la situación no cambió excesivamente en las primeras décadas del siglo XX, ya que el número de viajeros embarcados o desembarcados con motivos puramente turísticos en los puertos españoles siguió siendo muy escaso. Y es que resulta obvio que en Barcelona, y a pesar del impulso de organizaciones como la Sociedad de Atracción de Forasteros (Garay y Cànoves, 2009), la situación social del momento no favorecía precisamente la llegada del tipo de turista crucerista.

El estallido de la Guerra Civil y la posterior posguerra perpetuaron el escaso tránsito de viajeros por mar vinculados con el ocio. Durante la contienda, como también ocurrió en las guerras mundiales, muchos buques fueron utilizados con diferentes propósitos (transporte de armamento, hospitales móviles, cárceles incluso) y una gran parte de ellos fueron destruidos (Fernández Fúster, 1991). De hecho, no sería hasta pasada la posguerra, cuando una iniciativa un tanto peculiar reactivaría el escaso tránsito de cruceros en la ciudad. Fue en 1956 cuando la compañía Transmediterránea transformó uno de sus buques, el Ciudad de Toledo, en buque-exposición, presentando los avances de la industria española (un proyecto propagandístico vinculado al régimen franquista) en una travesía por 16 países americanos (Gómez Santos, 1991). Pocos años después, en 1961, otro buque de la compañía, el Villa de Madrid, realizó un crucero por el Mediterráneo con destino en el puerto griego del Pireo y con otro motivo excepcional: la boda de los Príncipes de Asturias. Mientras, el puerto de 
Barcelona, con un carácter eminentemente industrial, seguía creciendo al amparo de nuevos proyectos como el Plan General de Desarrollo de 1965-1966, extendiéndose hacia el delta del Llobregat, con la construcción de un puerto interior junto al río (Alemany, 2002).

Ya en los años setenta aparecerán los que se pueden considerar primeros servicios dedicados al turismo de cruceros. Es la compañía Ybarra, que con sus buques Cabo San Vicente y Cabo San Roque la que iniciará diferentes rutas turísticas, especialmente en dirección a Latinoamérica, aunque el producto todavía estaba pensado para un público de alto poder adquisitivo y que disponía de suficiente tiempo como para poder embarcarse en un viaje de larga duración. De hecho, a parte de la escasa demanda internacional que también llegaba en los setenta tan solo unos 20.000 españoles elegían un crucero para realizar sus vacaciones, permaneciendo esta cifra casi constante hasta mediados de los años noventa, en los que comenzó un crecimiento sostenido que lo llevaría a las cifras actuales, que superan los 300.000 cruceristas (Paniagua, 2005). Con la llegada de la democracia y los cambios políticos también se iban a producir cambios que afectarían al turismo de cruceros. Para empezar, en 1978 el puerto de Barcelona conseguía su Estatuto de Autonomía del Puerto, lo que significaba un paso más allá en cuanto a una gestión local del mismo, más eficiente y emprendedora. Y es precisamente en esta época en la que el Puerto de Barcelona se desarrolla a partir de una concepción funcional y se divide ya en tres zonas, el Port Vell (Puerto Viejo), el Puerto Comercial y el Puerto Logístico, ampliándose de nuevo sus infraestructuras hacia el sur con la compra de terrenos al Consorcio de la Zona Franca.

Será entre finales de los años setenta e inicios de los ochenta que aparecen en España nuevas compañías dedicadas al negocio del crucerismo como el turoperador Unión Lloyd, la compañía Central de Cruceros, surgida de los departamentos marítimos de diferentes agencias de viaje (Meliá, Marsans y Wagons Lit) o el turoperador Latitud 4. Con todo, todavía en la década de los ochenta del siglo XX Barcelona seguía recibiendo a un escaso número de turistas de cruceros, ya que no era un destino especialmente reconocido a nivel internacional y tenía aún menor proyección entre el turista elitista que todavía predominaba en esta tipología en la Europa de los años ochenta. A esto cabía sumar que Barcelona no era todavía un destino turístico cuyos recursos resultaran especialmente atractivos para el público en general. Con todo, algunas de las primeras experiencias de navieras españolas como Transmediterránea tuvieron la ciudad de Barcelona como puerto base durante estos años. A mediados de la década de los ochenta esta compañía decidía dar un paso más tras experimentar con viajes de corto trayecto (fines de semana con pequeñas escalas en las Islas Baleares) y realizar viajes de ocho días por el Mediterráneo con salida en la ciudad condal.

No obstante, todo iba a cambiar en breve a partir de la celebración de los XXV Juegos Olímpicos de Barcelona en julio de 1992. Alrededor de la preparación del evento se iba a movilizar toda la ciudad, la región y el país, acometiendo una profunda transformación de las infraestructuras y de la propia imagen de la ciudad. Y la zona portuaria y las actividades relacionadas no quedaron al margen. La apertura de la ciudad al mar y el hecho de que en el año 1987 se comenzaba a elaborar el Plan Estratégico de Barcelona se concretaron en un ambicioso proyecto que transformaría esta infraestructura y facilitaría la explosión del turismo de cruceros en los siguientes años. 


\section{1992, EL AÑO CLAVE PARA EL CRUCERISMO EN BARCELONA}

Hoy en día no cabe duda que los Juegos Olímpicos de 1992 marcaron un antes y un después en el desarrollo de las diferentes tipologías turísticas en Barcelona. El turismo de cruceros no ha sido una excepción. Los Juegos significaron una prueba de fuego para la gestión del puerto de Barcelona, que estuvo a la altura del evento acogiendo en sus instalaciones un buen número de grandes cruceros que sirvieron de hoteles flotantes durante el acontecimiento.

En todo caso Barcelona tenía ya una dilatada experiencia en relación a la celebración de grandes eventos, iniciada con la celebración de la Exposición Universal de Barcelona, continuada con la de 1929 y con otros importantes hitos como los Mundiales de 1982. La elección de Barcelona como sede de los XXV Juegos Olímpicos llevó al consistorio municipal a un nuevo proceso de reflexión, en el que se buscaba una profunda transformación de la ciudad y donde el turismo debía ser uno de los ejes centrales del desarrollo futuro de la misma. Con este propósito se iniciaron ya en 1987 las primeras reuniones entre el Ayuntamiento, representado por el Patronato Municipal de Turismo, y el Comité de Turismo de la Cámara de Comercio, Industria y Navegación de la ciudad. Se iniciaba así una colaboración que daría como fruto el Plan Estratégico, en el que se consideraba de forma muy especial el potencial de la urbe para ser un importante destino turístico internacional en el futuro, aprovechando el impacto que debería suponer la celebración de los Juegos (Duran, 2002).

El Plan tuvo una primera etapa entre 1989 y 1991 dedicada al análisis y la diagnosis de la situación turística de la ciudad y concluyó con la creación en 1993 de un organismo de promoción turística, Turisme de Barcelona, cuya responsabilidad en el despegue de la ciudad como destino es innegable, especialmente en relación a su labor en cuanto a la inversión en la promoción turística y el branding de la ciudad. Con la definición del Plan y la posterior creación de Turisme de Barcelona se pasó de una etapa en la que se promocionaba Barcelona de forma genérica, a una nueva centrada en la que los objetivos eran la promoción específica y la especialización a partir de la identificación de diferentes segmentos de mercado, entre los que se encontraba el mercado del turismo de cruceros.

Hoy en día es indiscutible que la imagen proyectada al mundo a partir de este evento sirvió para transformar la visión de Barcelona, de una ciudad vista como un centro industrial con un turismo mayoritariamente de negocios (un 70\% en 1990) a un destino donde el peso del turismo vacacional es hoy en día mayoritario (aunque sigue siendo importante el segmento de negocios) (Duran, 2002). Esto sin tener en cuenta que la ciudad también se ha convertido en uno de los principales destinos del turismo de conferencias y convenciones, con diferentes eventos (como el World Mobile Congress) de relevancia internacional, o que es uno de los principales destinos en cuanto a eventos culturales (Sónar) o deportivos (como el torneo Conde de Godó o las diferentes carreras de atletismo que se realizan de manera regular a lo largo del año) y que también ofrece una amplia oferta de turismo de sol y playa en sus diferentes playas y en las localidades cercanas.

Incluido en este amplio grupo de nuevos productos y nuevos mercados de éxito en la ciudad se encuentra sin lugar a dudas el turismo de cruceros. De hecho el Plan Estratégico mencionado percibió desde un principio a esta tipología como un elemento clave, creando un plan específico que debería impulsar a Barcelona como el principal destino de cruceros 
en el Mediterráneo. El trabajo se iniciaba considerando la capacidad de alojamiento de los cruceros como apoyo a los hoteles de la ciudad para la acogida durante los Juegos. Se trató de una prueba arriesgada, que ponía a prueba la gestión de los grandes cruceros en unas instalaciones portuarias un tanto deficientes. Finalmente, a pesar de los riesgos, la experiencia fue un éxito y durante la celebración de los Juegos once buques ofrecieron sus servicios en la ciudad (Castejón, 1998), convirtiéndose en la segunda gran concentración de alojamiento, tras la Villa Olímpica. El resultado fue una logística adecuada con un correcto funcionamiento, lo que demostró la capacidad organizativa de los gestores del puerto y evidenció la potencialidad de Barcelona para promover el crucerismo.

Así mismo, tan importante como la propia estrategia público-privada en cuanto a la potenciación del turismo resultó el conjunto de actuaciones urbanísticas llevadas a cabo en paralelo a la preparación de los Juegos. Con éstas la trama urbana se abrió al mar después de décadas de darle la espalda, especialmente a través de la remodelación del Port Vell, la construcción de los nuevos barrios marítimos y la creación de nuevos espacios públicos (playas y paseos) en el litoral, y los cambios se extenderían a la principal infraestructura que necesitaba este tipo de turismo, el puerto. Resulta interesante en este sentido que justo en 1992 fueran suprimidas en España las juntas de obras y puertos autónomos, creándose las autoridades portuarias. Así es como el Port de Barcelona se rebautiza como Autoritat Portuaria de Barcelona, naciendo una institución cuyo papel ha sido clave para entender el desarrollo de las infraestructuras portuarias, especialmente importante respecto a la posibilidad de acoger la creciente demanda de turistas que ha tenido lugar.

En este sentido, a partir de 1992 los dos elementos comentados se sumarían para dar lugar al éxito del turismo de cruceros que hoy en día conocemos. Por un lado, el papel de Turisme de Barcelona en la proyección de Barcelona como un interesante destino urbano a nivel internacional, recibiendo un creciente número de visitantes $\mathrm{y}$, por otro lado, el papel de la Autoritat Portuaria en la adaptación del puerto de Barcelona y su relación con las organizaciones navieras, han sido claves para poder acoger el desarrollo de la nueva corriente de turistas, los cruceristas, que se ha sumado a otros segmentos de demanda cuyo crecimiento ha sido espectacular en las dos últimas décadas.

\section{LA APARICIÓN DE NUEVOS MERCADOS E INFRAESTRUCTURAS PARA EL CRUCERISMO EN BARCELONA}

El éxito del turismo en la Barcelona post-olímpica es un ejemplo de cómo se ha sabido rentabilizar y proyectar la marca de ciudad gracias al evento. En los noventa, y con la breve excepción de los años de crisis inmediatamente posteriores a los Juegos, la llegada de turistas a la ciudad creció de manera considerable. Entre el año 1991 y el 2005 el número de turistas que pernoctaban en la ciudad prácticamente se triplicó, pasando de poco más de un millón setecientos mil a más de cinco millones según el Ayuntamiento de Barcelona (AB, 19922011). Diferentes elementos jugaron a su favor, como el propio desarrollo de la oferta, que creció más de un 30\% (AB, 1992-2011), los importantes cambios asociados a la transformación de la demanda, con el impacto de las tecnologías de la información y la comunicación (TIC) o el aprovechamiento de su renta de situación ampliado por el avance de las compañías aéreas de bajo coste. Barcelona tiene además la ventaja de situarse en el centro de un des- 
tino tradicional de sol y playa, el conformado por el litoral catalán. Después de décadas de relativo abandono, el patrimonio natural de la ciudad (especialmente las playas) y también el cultural se revitalizaba y se ponía en valor para un público internacional que redescubría sus atractivos tras años de letargo.

Barcelona no solo se sitúa a escasa distancia de los grandes mercados europeos (acortada con la proliferación de las compañías aéreas de bajo coste), sino que además está localizada en la cuenca mediterránea, con todo lo que ello significa en cuanto a la posibilidad de convertirla en centro de desplazamientos hacia numerosos destinos que cuentan con espectaculares recursos tanto naturales, como culturales y que se encuentran a una noche de navegación. Ello significa que ofrece amplias posibilidades de ser un destino que complementa mar y tierra como pocos. Teniendo en cuenta estos elementos y tras el éxito de la experiencia olímpica, los gestores de la Autoritat Portuaria de Barcelona entendieron las potencialidades que ofrecía la ciudad para despegar en el turismo de cruceros, impulsando un tráfico de pasajeros que en principio no debía suponer un incremento importante en inversiones en infraestructuras y que a cambio podía reportar notables beneficios. Fue con esta intención que se configuró un Plan Especial, consensuado entre la administración municipal y la Autoritat Portuaria con el objetivo de proceder a una reordenación del Port Vell, previendo la localización de nuevas terminales para líneas regulares y cruceros (Castejón, 1998).

Con el incremento de las escalas de cruceros en la ciudad entraría en funcionamiento en el verano de 1994 la primera Terminal A, al año siguiente, en verano de 1995, se inauguraba la Estación Marítima Internacional, y al finalizar la década de los noventa el puerto de Barcelona contaba ya con cinco estaciones marítimas para cruceros y dos que se podían intercambiar con los ferries. Las cinco terminales exclusivas (A y B del muelle adosado, la del Port Vell y las dos del muelle de Barcelona) ofrecían cerca de 2.000 metros de línea de atraque y cerca de 15.000 metros cuadrados de áreas construidas para el servicio a los buques. El puerto de Barcelona se había convertido así en la infraestructura más completa para cruceros de España.

En todo caso el éxito de estos años se debe en gran medida a la conjunción exitosa del trabajo entre la Autoritat Portuaria y Turisme de Barcelona. Mientras la Autoritat Portuaria se centraba en atender a los armadores y tripulaciones como clientes, Turisme de Barcelona se ocupaba de desarrollar los ámbitos de la promoción y comunicación turística. Así, Turisme de Barcelona se preocupaba por la imagen de la ciudad, promoviendo la llegada de turistas y focalizaba su promoción exterior en las agencias de viajes internacionales, especialmente norteamericanas, mientras que la Autoritat Portuaria se encargaba de promocionar el crucerismo en las ferias internacionales especializadas (como ejemplo, la Seatrade Mediterranean Cruise and Ferry Convention, que ya se celebraba en Barcelona a mediados de los noventa o la reunión de META, la Mediterranean Travel Association, que se ha congregado por primera vez en Barcelona en 2007).

Gracias a la acción conjunta de Turisme de Barcelona y la Autoritat Portuaria, Barcelona se ha convertido en el principal puerto base de cruceristas (puerto de inicio y finalización de trayectos) del Mediterráneo. Otros elementos claves han favorecido este crecimiento del crucerismo y cabe destacar el disponer de unas instalaciones de embarque óptimas y seguras, infraestructuras relacionadas con el ocio y especialmente una buena conexión (relación inter-modal) con otros medios de transporte. Por ello el hecho de que el aeropuerto del Prat 
está bien conectado con la zona portuaria, a menos de media hora de distancia resulta muy atractivo para los cruceristas que toman como lugar de embarque Barcelona. En este tipo de decisiones intervienen además otros factores como los propios mercados, los precios competitivos así como la habilidad de las compañías de intermediación para organizar la red de ventas. Sin embargo, todo ello no sería suficiente sin contar con el atractivo de la ciudad como destino internacional (Montero, 1996).

Gráfico 1

DEMANDA DE TURISMO DE CRUCEROS EN BARCELONA, 1992-2010

Número total de pasajeros en cruceros (embarcados, desembarcados y en tránsito)

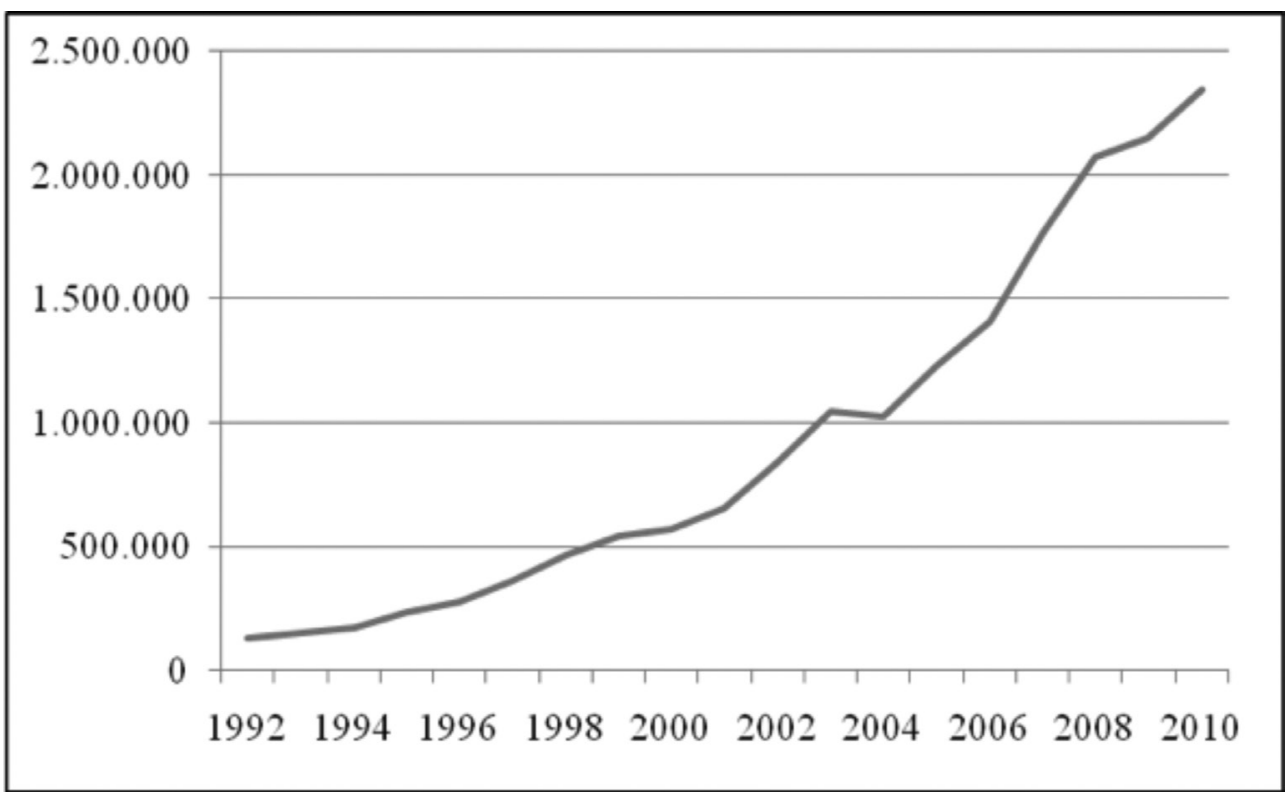

Fuente: Elaboración propia a partir de datos de la Autoritat Portuaria de Barcelona cedidos al Ayuntamiento de Barcelona (AB, 1992-2011)

Este desarrollo de la oferta de instalaciones y de la promoción de Barcelona como destino ha tenido su traducción en un desarrollo espectacular de la demanda, como se puede apreciar en el Gráfico 1. En el mismo se refleja el notable crecimiento del número de pasajeros en cruceros en la ciudad, que en el periodo analizado muestra un promedio cercano al $18 \%$ de crecimiento, con algunos ejercicios donde la tasa de crecimiento interanual se situó cercana incluso al 30\%. Resulta además interesante saber que según la OMT (2008) muchos de estos viajeros son repetidores; de hecho entre un 40 y un 60\%, una cifra realmente alta y prácticamente insólita en otras tipologías. Teniendo en cuenta que según las estadísticas oficiales de Puertos del Estado, el crecimiento de la demanda en el período 2002-2009 en el conjunto de puertos españoles ha sido elevado (un $12 \%$ en los ocho últimos años, desde los que se dispone de estadísticas fiables) y teniendo en cuenta que el crecimiento a nivel internacional 
se sitúa en torno al $8 \%$ para el mismo período, podemos apreciar como el caso Barcelona ha sido realmente único en los últimos años.

Siguiendo con la perspectiva de la demanda cabe decir que además del propio atractivo de la ciudad, su correcta promoción y la mejora de los servicios portuarios en los últimos años, su despegue como destino crucerista ha coincidido afortunadamente en el tiempo con dos elementos que le repercuten directamente: el desarrollo del Mediterráneo como destino (ya que Barcelona es puerto base de itinerarios) y el del mercado europeo (ya que la ciudad se beneficia especialmente del crecimiento de este mercado). Respecto al Mediterráneo como destino, su desarrollo ha sido posterior en el tiempo en relación a otros destinos, especialmente la zona del Caribe, cuya evolución se inició ya en los sesenta. En los últimos años su cuota no ha hecho más que crecer, especialmente en la estación estival, en la que según la OMT (2008) alcanza ya el 30\% del total internacional (mientras que en invierno supone solo un 5\%). Con todo, se trata de un destino que todavía tiene perspectivas de crecimiento, ya sea en la temporada de invierno como especialmente en relación a la posibilidad de incorporar nuevos destinos locales en el Mediterráneo Oriental y Norte de África, zonas que habitualmente han presentado problemas de seguridad y/o calidad pero que pueden en el futuro aportar importantes recursos y productos turísticos (ya es así en el caso de Grecia y Turquía).

De la misma manera que el desarrollo del Mediterráneo como destino crucerista afecta positivamente al propio desarrollo de Barcelona como puerto base, también es importante el desarrollo de los propios mercados europeos. En este contexto, el turismo de cruceros acusó una excesiva dependencia de la demanda norteamericana hasta bien entrados los años noventa, en los que entró en liza el mercado británico, especialmente a partir de 1996 cuando aparecieron en el negocio nuevos operadores turísticos, especialmente británicos. Hacia finales de la década de los noventa el crecimiento de la demanda vino dado por la consolidación de las navieras europeas y la irrupción de las compañías norteamericanas. En los inicios del el siglo XXI se incorpora un nuevo elemento: el crecimiento de la demanda de la Europa continental (Alemania especialmente), que en poco tiempo llegará a superar la cuota británica, como se aprecia en la Tabla 1.

Por lo que respecta al mercado español, el año 2001 fue un año de verdadera inflexión. Tras décadas de presentar datos realmente circunstanciales, la demanda de turismo de cruceros empezó a crecer a un ritmo realmente importante. En concreto, se estima que el mercado español creció alrededor de un $40 \%$ entre 1999 y 2004, siendo el mercado de mayor crecimiento en toda Europa (Paniagua, 2005). Este desarrollo se ha basado especialmente en la importante inversión realizada por las compañías nacionales (Royal Hispania, Spanish Cruise Line, Pullmantur Cruceros, Globalia) junto con el desembarco de las grandes compañías transnacionales, pero también por el propio desarrollo de la demanda turística nacional. En este sentido, el futuro de las compañías españolas pasa por el aprovechamiento de la madurez del turista de cruceros español a través de una especialización de los operadores en destinos o producto y también a partir de la entrada como operadores globales en nuevos mercados, especialmente Sudamérica. En Barcelona, tras la finalización de los Juegos Olímpicos diferentes buques de estas compañías han estado operando, dedicándose total o parcialmente al mercado español. 


\section{BARCELONA, UNO DE LOS PRIMEROS DESTINOS CRUCERISTAS INTERNACIONALES}

Llegando ya a la actualidad, es indudable que el turismo de cruceros es una de las tipologías y mercados de mayor crecimiento en el mundo. Sus características le convierten en un buen ejemplo de nuevo turismo, al menos por lo que respecta a gran parte de la configuración del producto, desde la vertiente de la oferta. Tal y como indica la Cámara de Comercio de Valencia (CCV, 2009) en su estudio al respecto, esta oferta presenta diferentes atractivos entre los que se podrían destacar su configuración multi-destino (diferentes destinos a visitar en el mismo viaje), el hecho de incluir un hotel móvil (comodidad y avance de tiempo), la fórmula de todo incluido (servicios incluidos en el buque) o la flexibilidad, comodidad y seguridad del desplazamiento. Este mercado resulta además cada vez más interesante para muchos destinos, especialmente grandes ciudades portuarias, ya que también genera importantes ingresos durante la visita de los turistas en la ciudad de atraque. El perfil medio del crucerista es el de un turista de mediana edad (alrededor de 45 años) y con ingresos medioaltos. En los últimos años también ha crecido el segmento más joven, aunque especialmente se ha notado la incorporación del mercado familiar.

Y es en este contexto que Barcelona se ha convertido en un destino líder del turismo de cruceros a nivel internacional. La clave del éxito de Barcelona se explica por la inteligente promoción del destino, que arranca la época de las Olimpiadas, una eficiente colaboración entre las autoridades, las inversiones necesarias cuanto a la infraestructura portuaria, la seguridad del destino (especialmente si se compara con el Mediterráneo Oriental) y el desarrollo de nuevos productos relacionados a partir de una buena segmentación de la demanda.

Centrándonos en esta demanda, en el año 2010 y a pesar de la crisis, se batió un nuevo récord en la llegada de viajeros con más de 2.300.000 pasajeros de cruceros en la ciudad. Barcelona representa cerca de un $40 \%$ del movimiento crucerista en los puertos españoles (tal como muestra la tabla 1). Además, para el ejercicio 2011 la Autoritat Portuaria espera superar las 900 escalas y llegar a los 2,5 millones de cruceristas, especialmente gracias a la llegada de nuevos buques con mayor capacidad. Cabe señalar que el sector ha estado preocupado recientemente por el importante crecimiento en el precio del combustible, pero justamente Barcelona es uno de los puertos mejor localizados para sortear en gran medida este inconveniente, dada su cercanía a otros muchos puertos turísticos del Mediterráneo Occidental. Gracias a estos elementos, la mayor parte de las navieras siguen confiando en el Puerto de Barcelona y está previsto que para los próximos años mantengan las escalas y sigan utilizando a la ciudad como puerto base.

También en relación a la demanda y sus repercusiones, los principales atractivos para el turista de cruceros en la ciudad (con un alto porcentaje de repetidores), son el arte, la arquitectura, la historia, el comercio, la gastronomía, un transporte público correcto y la seguridad de la misma (óptima si la comparamos con otros destinos de la zona). Es importante señalar que el turismo de cruceros no ha supuesto una desventaja para la hotelería de la ciudad, especialmente porque Barcelona es un puerto base y en ese sentido lo que supone es una oportunidad para la misma, ya que muchos turistas pernoctan algunos días antes de embarcar o justo al acabar el viaje. De hecho, en los últimos años en los que el crecimiento del turismo de cruceros ha sido tan importante, el crecimiento de turistas y pernoctaciones también ha sido notable a pesar del contexto de crisis (un promedio de un 5\% y un 4\% entre 2005 y 2009 
para turistas y pernoctaciones, respectivamente, según datos del ayuntamiento de la ciudad) y la capacidad hotelera no se ha resentido, sino al contrario ha continuado creciendo tanto en nuevos establecimientos como en pernoctaciones.

En relación al impacto de este tipo de turismo en la ciudad, según la OMT (2008) se estimaba que en el año 2006 este ascendía a más de 150 millones de euros, teniendo en cuenta un gasto medio por día y turista de 90 euros y un precio medio por habitación hotelera de 115 euros. En este sentido, se consideraba que un $82 \%$ de los cruceristas no pernoctaban, pero que un $11 \%$ lo hacían una noche, un $5 \%$ dos noches y un $2 \%$ tres noches. También en el año 2006 la European Cruise Council junto con Euroyards, MedCruise y Cruise Europe tomaron la decisión de realizar un estudio (ECC, 2007) sobre el impacto de los cruceros en las economías locales, con el fin de hacerlo llegar a las administraciones locales, nacionales y regionales europeas. Éste indicaba que en Barcelona el negocio de los cruceros estaría siendo responsable de 14.000 puestos de trabajo, con una remuneración total superior a los 400 millones de euros. En todo caso, la misma Autoritat Portuaria considera que teniendo en cuenta que los cruceros ocupan entre un 10 y un $15 \%$ de la línea de atraque del puerto tan solo generan un $3,5 \%$ de la cifra de negocio del puerto, con lo que la mayor parte de sus beneficios no repercuten en esta infraestructura sino en la propia ciudad, con lo que parece demostrada la simbiosis existente entre la dinámica de la misma y esta tipología turística.

En relación a las perspectivas de futuro, todo indica que el Puerto de Barcelona tiene en los próximos años un importante potencial para crecer ya que todavía existe un mercado objetivo muy amplio, especialmente en Europa, que actualmente solo ocupa una cuarta parte del volumen mundial de cruceristas. Aun teniendo en cuenta las diferencias a nivel internacional (ya sea por la localización, mercados, etcétera), la OMT entiende que el futuro del crucerismo en Barcelona pasa por el impulso de diferentes elementos. Entre estos elementos de futuro se pueden destacar, desde la perspectiva de la oferta, la necesidad de una mayor flexibilidad, la situación de oligopolio en la que puede incurrir el sector, la apuesta por buques cada vez mayores, el esfuerzo por la innovación y la mejora de los servicios, la creciente regulación en diferentes destinos y los temas relacionados con la seguridad. También resulta evidente que los aspectos relacionados con la sostenibilidad y la responsabilidad serán claves para entender la evolución del negocio. En cuanto a la demanda, los cambios socio-demográficos (envejecimiento de la población, diferentes unidades familiares, segmentación de las vacaciones), el proceso de globalización, el cambio climático o el uso de las tecnologías de la información y la comunicación también jugarán un papel importante. Barcelona como destino internacional deberá estar atenta en cuanto a estos cambios para poder afrontar los riesgos y aprovechar las ventajas que aparecerán en estos nuevos escenarios.

\section{CONCLUSIONES}

En este trabajo se ha demostrado la importancia actual del turismo de cruceros para Barcelona y como éste ha pasado de una posición marginal en el período previo a la celebración de los Juegos Olímpicos de 1992 a situarse actualmente en una posición de liderazgo internacional. El trabajo ha mostrado también la importancia de los agentes locales en el impulso al desarrollo de esta tipología. La preparación de los Juegos fue fundamental tanto para la adaptación de las infraestructuras portuarias para el tráfico turístico y tanto o más relevante 
fue el papel del consorcio Turisme de Barcelona en la proyección de la ciudad como destino turístico en general y crucerista en particular.

La decidida implicación de dichos agentes se vio además beneficiada por un conjunto de elementos de oferta y demanda que han propiciado el espectacular crecimiento del crucerismo en las dos últimas décadas. Desde el punto de vista de la oferta, cabe destacar la óptima renta de situación de la ciudad de Barcelona. La ciudad se sitúa a escasa distancia de los principales puertos del Mediterráneo, próxima a los principales recursos turísticos del Mediterráneo y también a los principales mercados emisores europeos que se ven facilitados por las compañías aéreas de bajo coste. Todo ello apoyado por la excelente situación de las infraestructuras portuarias por lo que respecta a otros servicios de transporte a corta, media y larga distancia (transporte urbano, AVE, Aeropuerto) así como a los principales recursos de la ciudad. Finalmente también por su buena conexión con importantes zonas turísticas tradicionales como la Costa Brava y la Costa Dorada.

La ampliación y mejora de las infraestructuras cruceristas, junto con los adecuados servicios logísticos en la zona portuaria, ha facilitado el desembarco de las grandes compañías navieras, cada vez más poderosas en un contexto de concentración y globalización del sector. El elemento vinculado a la seguridad del destino también ha sido clave en un sector sensible a estos aspectos, máxime cuando gran parte del Mediterráneo Oriental aún está lejos de poder competir con el Occidental debido a una percepción de inseguridad. Además, el desarrollo crucerista también se alimenta de la amplia y diversa oferta hotelera en la ciudad, que siendo puerto base de gran número de trayectos cruceristas, facilita la pernoctación a muchos turistas que parten o regresan de los mismos. Por otro lado, aunque estamos hablando de una actividad con elementos representativos de una estructura de mercado específica, se reproducen patrones de los mercados más tradicionales (masificación, estacionalidad, concentración de la oferta y poder de las grandes compañías).

El artículo ha puesto de manifiesto que la demanda de turismo de cruceros en Barcelona ha crecido de manera espectacular en los últimos años, beneficiada por diferentes elementos que hacen atractivo a este tipo de turismo y al destino. Barcelona se sitúa en el cuarto destino de Europa y este crecimiento se sitúa además en un contexto de crecimiento del Mediterráneo como destino regional, en el que ha crecido notablemente el mercado norteamericano, necesitado de diversificación (el Caribe es ya un destino maduro), a la vez que se ha producido un importante crecimiento en los últimos años del mercado europeo, especialmente alemanes (restando peso al mercado británico, que era mayoritario) y mientras el mercado español experimentaba también un importante desarrollo desde el inicio del presente siglo. Hemos comprobado que el turismo de cruceros se ha popularizado y en gran medida ha abandonado sus características minoritarias y elitistas. Se ha beneficiado además de otros fenómenos, como el crecimiento del mercado de la Tercera Edad (más gente mayor con mayores ingresos y mayor disponibilidad de tiempo de ocio) así como de su capacidad para dar servicio a segmentos específicos, como el turismo familiar.

En definitiva, Barcelona es ya uno de los principales destinos cruceristas a nivel internacional, lo que puede añadir a su reconocido atractivo en otras tipologías. No obstante, para mantener esta posición los responsables de la promoción turística, las autoridades portuarias y las compañías que operan en la ciudad deberán estar atentos a los nuevos riesgos y oportunidades derivados del actual contexto social y económico. Entre éstos, se podrían 
destacar los aspectos vinculados con la seguridad, la necesidad constante de innovación en materia logística, los elementos relacionados con la necesidad de una mayor especialización del crucero que den respuesta a la creciente segmentación de la demanda y, finalmente, la mayor preocupación de la demanda por los aspectos relacionados con la sostenibilidad y la responsabilidad social.

\section{AGRADECIMIENTOS}

Los autores agradecen al editor y a los evaluadores del artículo sus comentarios, críticas y sugerencias. Parte de esta investigación ha sido posible gracias a la financiación proporcionada por la CICYT (proyecto CSO2009-11793).

\section{BIBLIOGRAFÍA}

AB (1992-2011): Anuari Estadístic de la ciutat de Barcelona. Barcelona. Ajuntament de Barcelona.

ALEMANY, J. (2002): El port de Barcelona. Un passat, un futur. Barcelona. Port de Barcelona.

ANDRIOTIS, K. y G. AGIOMIRGIANAKIS (2010): «Cruise visitors’ experience in a Mediterranean port of call». International Journal of Tourism Research, $\mathrm{n}^{\circ}$ 12(4), 390-404.

APB (2006-2009): Memoria corporativa. Barcelona. Autoritat Portuaria de Barcelona.

BRIDA, J.G., RIAÑO, E. y ZAPATA, S. (2012): «Percepciones de los residentes acerca de los impactos del turismo de cruceros en la comunidad: un análisis factorial y de clústeres». Cuadernos de Turismo, no 29, 79-107.

BUTLER, M. (2001): Análisis económico y oportunidades para España en el mercado de los cruceros turísticos. Madrid. Universidad Politécnica.

BUZARD, J. (1993): The beaten track: European tourism, literature, and the ways to culture, 1800-1918. Oxford. Oxford University Press.

CASTEJÓN, R. (1998): El tráfico de grandes cruceros en Barcelona. Un nuevo elemento de relación entre el puerto y la ciudad. Le Havre. Association internationale villes \& ports.

CCV (2009): El turismo de cruceros. Valencia. Cámara de Comercio, Industria y Navegación de Valencia.

CLIA (2005): CLIA's 2004 Cruise Market Profile: Report of Findings. Fort Lauderdale. Cruise Lines International Association.

DRISDALE, J. (1997): «Evolution of the cruise market in the Mediterranean and Easter Atlantic». Portnewspaper, $\mathrm{n}^{\circ} 116,11-12$.

DURAN, P. (2002): «The impact of the Olympic Games on tourism. Barcelona: the legacy of the Games 1992-2002» en Barcelona: l'herència dels Jocs. 1992-2002 (Moragas, M. y Botella, M.). Barcelona, Planeta y Ajuntament de Barcelona.

ECC (2007): Contribution of Cruise Tourism to the Economies of Europe. Brussels. European Cruise Council.

ESTEVE, R. (1998): «El turismo de cruceros» en La Actividad Turística Española (VV. AA.). Madrid, Asociación Española de Expertos Científicos. 627-636. 
FERNÁNDEZ FÚSTER, L. (1991): Historia general del turismo de masas. Madrid. Alianza Editorial.

GARAY, L. A. y G. CÀNOVES (2009): «El desarrollo turístico en Cataluña en los dos últimos siglos: una perspectiva transversal». Documents d'Anàlisi Geogràfica, nº 53, 29-46.

GÓMEZ SANTOS, M. (1991): Todo Avante. Madrid. Compañía Trasmediterránea.

HALL, J.A. y R. BRAITHWAITE (1990): «Caribbean cruise tourism: A business of transnational partnerships». Tourism Management, $\mathrm{n}^{\circ}$ 11(4), 339-347.

JOHNSON, D. (2002): «Environmentally sustainable cruise tourism: a reality check». Marine Policy, $\mathrm{n}^{\circ}$ 26(4), 261-270.

LAWTON, L. J. y R. BUTLER (1987): «Cruise ship industry-patterns in the Caribbean 1880-1986». Tourism Management, $\mathrm{n}^{\circ}$ 8(4), 329-343.

MONTERO, L. (1996): «Estrategias de los puertos base de cruceros en el Mediterráneo». Boletín de Puertos del Estado, n 35, 16-20.

MURIAS, R. (2002): La industria del crucero en el siglo XXI. Implicación en los puertos españoles y perspectivas de futuro. Barcelona. Universitat Politèncica de Catalunuya.

OMT (2003): La actividad de los cruceros turísticos en el mundo. Madrid. Organización Mundial del Turismo.

OMT (2008): Turismo de cruceros: situación actual y tendencias. Madrid. Organización Mundial del Turismo.

PANIAGUA, A. (2005): «El mercado de los cruceros en España. Una perspectiva histórica (1994-2004)». Estudios turísticos, n $165,131-160$.

TOWNER, J. (1985): «The grand tour: A key phase in the history of tourism». Annals of Tourism Research, $\mathrm{n}^{\circ}$ 12(3), 297-333.

WOOD, R. E. (2000): «Caribbean cruise tourism:: Globalization at sea». Annals of Tourism Research, $\mathrm{n}^{\circ} 27(2), 345-370$. 
